\title{
Biomechanical behaviour and hyperelastic model parameters identification of sheep omasum
}

\section{Lebohang Lebea}

University of South Africa https://orcid.org/0000-0001-8076-1536

\section{Harry Ngwangwa}

University of South Africa https://orcid.org/0000-0001-5486-8049

Thanyani Pandelani

University of South Africa https://orcid.org/0000-0003-2107-5194

Fulufhelo Nemavhola ( $\nabla$ masitfj@unisa.ac.za )

University of South Africa https://orcid.org/0000-0002-6250-5157

\section{Research Article}

Keywords: Soft tissue mechanics, hyperelastic models, hyperelastic fitting, sheep omasum

Posted Date: October 26th, 2021

DOI: https://doi.org/10.21203/rs.3.rs-1015547/v1

License: (9) This work is licensed under a Creative Commons Attribution 4.0 International License.

Read Full License 


\title{
Biomechanical behaviour and hyperelastic model parameters identification of sheep omasum
}

\author{
Lebogang Lebea ${ }^{a}$; Harry Ngwangwa ${ }^{a}$, Thanyani Pandelania,b, Fulufhelo \\ Nemavhola ${ }^{a^{*}}$ \\ aUnisa Biomedical Engineering Research Group, Department of Mechanical Engineering, School of Engineering, \\ College of Science Engineering and Technology, University of South Africa, Pretoria, 0001, South Africa \\ ${ }^{b}$ Defence and Security, Council for Scientific and Industrial and Research, Pretoria, 001 \\ *Correspondence: masitfi@unisa.ac.za ; Tel.: (+27 (011) 471 2765)
}

\begin{abstract}
The function of the omasum is incompletely understood; however, the omasum plays an important role in the transport of appropriately sized feed particles from the reticulorumen to the abomasum, oesophageal groove closure, fermentation of ingesta, and absorption of water, volatile fatty acids, and minerals. The aim of this study is to evaluate the suitable hyperelastic anisotropic model based on biomechanical properties of sheep omasum. The results show that all five (5) hyperelastic models may be suitable for the evaluation of sheep omasum. The average coefficient of determination $\left(R^{2}\right)$ of Fung, Polynomial (Anisotropic), Holzapfel (2000), Holzapfel (2005) and Four-Fiber-Family hyperelastic models were found to be $0.79 \pm 0.19,0.95$ $\pm 0.05,0.92 \pm 0.07,0.93 \pm 0.05$ and $0.94 \pm 0.03$, respectively. Also, it was found that the best hyperelastic model for fitting uniaxial data of the sheep omasum was Polynomial (Anisotropic) with El of 100.0 followed by the Four-Fiber-Family model with El of 96.18 .
\end{abstract}

Keywords: Soft tissue mechanics; hyperelastic models; hyperelastic fitting; sheep omasum 


\section{Introduction}

Ruminant digestive physiology is largely dependent on pregastric fermentation in the forestomach. Therefore, in contrast to monogastric species the ruminant stomach system consists of a non-secretory forestomach functioning as a specialized mixing and fermentation vat and an acid and pepsinogen-secreting compartment functioning as stomach in the classical sense, the abomasum. The forestomach system anatomically consists of three primary structures: rumen, reticulum, and omasum[1]. Tumours of the omasum, reticulum, and rumen occur most often in cattle but are uncommon in most parts of the world. Papilloma, fibro papilloma, and fibroma are the most common tumours in the forestomach of cattle [2] .

There are many challenges that the field of tissue engineering must overcome to realise its clinical promise. These include concerns related to infection, adverse immune response, biochemical elements, and mechanical performance [3]. A biomechanical approach can be useful to investigate the stomach mechanical functionality [4]. The development of a computational model of the stomach can allow to examine in depth its functioning, related with complex neuro-gastrointestinal phenomena [5]. Models definition requires experimentations to characterise the organ geometrical conformation and the tissues mechanical behavior as such it is paramount to investigate the mechanical properties of omasum [6].

Computational modelling remains useful modality to validate experimental data and the mechanical behaviour of soft tissues [7-11]. However, to study the behaviour of these models it is essential to develop constitute model [12-16]. The Fung model is a widely utilised hyperelastic model which was originally developed for artery tissues. This hyperelastic model uses the strain energy function that depends on exponential function. Recently, a comparative study was presented for unfilled and highly filled rubbers using hyperelastic constitutive models [17]. In previous work, determination of layer-specific mechanical properties of human coronary arteries using Holzapfel constitute model was carried out [18-20]. The to develop these models the use of curve fitting is paramount [12]. Uniaxial testing remains the most common modality of mechanical analysis for biological and other soft materials [19]. It has been observed that Specimen mounting presents one of the most challenging tasks during soft tissue testing due to the fact that there is no widely accepted universal standard for clamp design for soft tissues. As a result there are many techniques that have been used to ensure that appropriate boundary conditions are imposed during soft tissue testing [21].

Study of mechanics of soft tissue is still relevant especially for the possible development of accurate computational models $[7,8,11,12,16,22-26]$ to study how diseases develop and advances. Sheep have been previous utilised to study mechanical properties of various important organs [12]. Currently there is no mechanical data on the sheep omasum. Therefore, this study aims to understand the mechanical properties of sheep omasum under uniaxial loading. Also, various hyperelastic models like Fung, Polynomial (Anisotropic), Holzapfel (2000), Holzapfel (2005) and Four-Fiber-Family were fitted in the biomechanical data to evaluate the best model that may be utilised for the development of computational models. 


\section{Experimental methods}

\subsection{Tissue acquisition}

The fresh sheep stomach was collected from the local abattoir. The stomach were then placed in the cooler box before transportation to the Biomechanics Lab for preparation and testing. The omasum was then isolated and taken out from the stomach.

\subsection{Uniaxial mechanical testing}

Twelve $(N=12)$ specimen of the omasum was cut out from the sheep stomach from an unknown age, weight, and pre-stomach disease. The sheep omasum were delivered from the local abattoir four hours after the slaughter. The delivery was done in a temperature-controlled bag at between $0^{\circ} \mathrm{C}$ and $3^{\circ} \mathrm{C}$. On arrival the temperature on the bag was measured to confirm and to ensure that mechanical properties were not compromised. The uniaxial testing was performed on the sheep strip of tissue (See Figure 1). Immediately after the arrival of the specimens, the sheep stomach were dissected to extract the selected omasum and thereafter stored in a $0.9 \% \mathrm{NaCl}$ physiological saline solution (PSS) for 20 minutes before mechanical testing. A total of 12 uniaxial tests were done on the omasum. During testing there were minimal challenges like tearing of tissue, fixation movement and tissue rapturing prematurely. The dissected omasum was then mechanically tested using CellScale UStretch (Watreloo, Canada) with a load cell of $44 \mathrm{~N}$ and force accuracy of $0.2 \%$ of the load capacity. The specimens were deposited in a bath filled with $0.9 \% \mathrm{NaCl}$ physiological saline solution. Each sample was immersed for four minutes before actual testing happened. To minimise the effect of strain measurements, the needles of the BioRake were inserted at a minimum of $0.2 \mathrm{~mm}$ from the edge. 


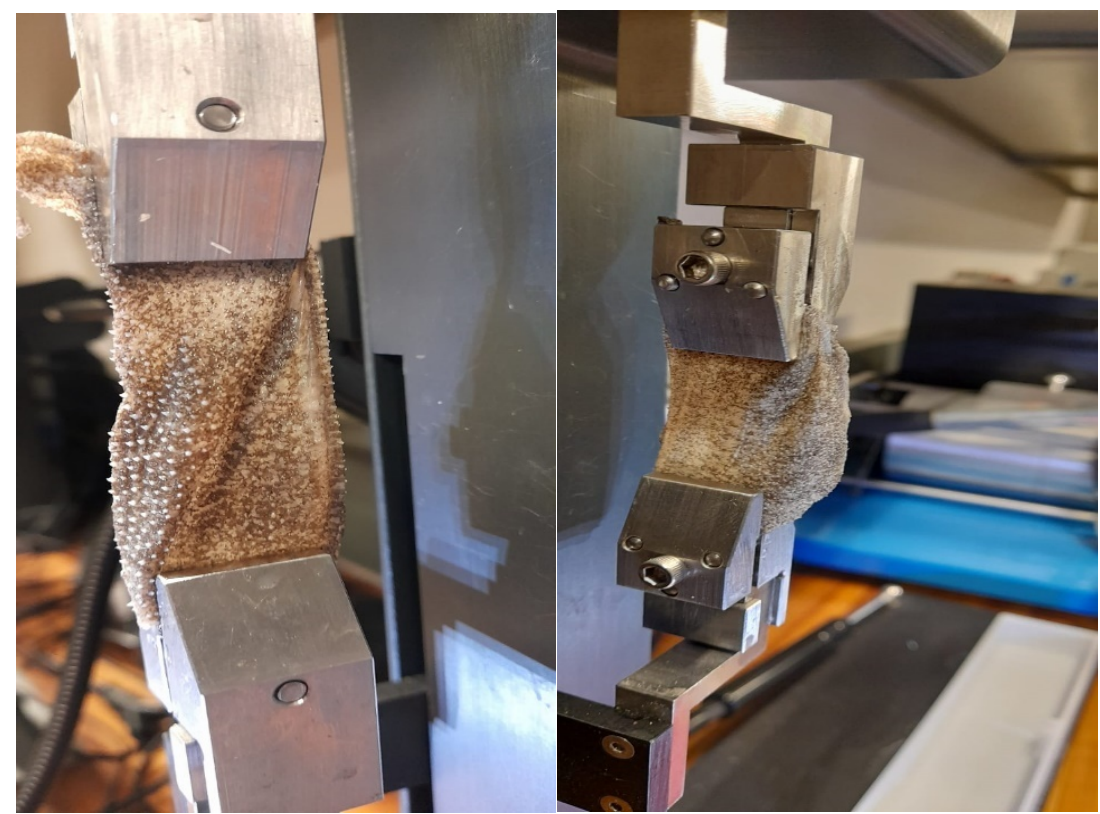

Figure 1: Experimental set-up of uniaxial testing of sheep omasum

\subsection{Tissue stress-stretch analysis}

The engineering stress and strain is determined from the force and displacement obtained during elongation of the muscle fibre of the sheep during uniaxial testing.

$\sigma_{i}=\frac{F_{i}}{A_{i}}$

$\varepsilon_{i}=\frac{\Delta l}{l_{o}}$

$A_{i}=t \times l_{h}$

Where:

$A_{i}-$ is the cross sectional area of the sheep muscle fibre

$\Delta l-$ is the change in length

$l_{o}-$ Original length

$\sigma_{i}-$ Engineering stress

$\varepsilon_{i}$ - Engineering strain?

$t$ - average thickness of the muscle fibre

$l_{h}$ - Breadth of the muscle fibre

\subsection{Evaluation index (EI) of the capability of anisotropic hyperelastic models}

To magnify the difference between coefficient of determination $\left(R^{2}\right)$ values, the evaluation index $(\mathrm{El})$ that can assess which models are better than the others was defined [13]. 
Evaluation Index $(E I)=\left[\frac{r-r_{\text {minimum }}}{r_{\text {maximum }}-r_{\text {minimum }}}\right]$

(4)

$r=a b s\left[\log _{10}\left(1-R^{2}\right)\right]$

(5)

Where $r$ is the quantity that is based on coefficient of determination $\left(R^{2}\right)$ and is expressed in Equation (5). The $r_{\text {minimum }}$ and $r_{\text {maximum }}$ values are minimum $r$ value for worst hyperelastic model and maximum $r$ value for best hyperelastic model, respectively. When $\mathrm{El}$ is 100 then it means that the hyperelastic model is the best. In addition, the greater the coefficient of determination $\left(R^{2}\right)$ the greater the El value.

\subsection{Selected hyperelastic anisotropic constitutive models}

To develop computational models for simulation, the material parameters from hyperplastic constitutive models are required. These parameters are normally utilised to predict the mechanical response of the omasum of the sheep. In this study, Fung, Polynomial (Anisotropic), Holzapfel (2000), Holzapfel (2005) and Four-fibre Family models were selected. The selected hyperelastic constitutive models were fitted using Hyperfit@ [27].

\subsubsection{Fung model}

The Fung model is a hyperelastic anisotropic material model proposed by Fung et al [28] for the stress-strain description of the arterial wall. The model is fully phenomenological and formulated through the components of Green-Lagrange strain tensor in cylindrical polar coordinates of the artery (R-radial, - circumferential, Z-axial). Exponential stress-strain behaviour is a characteristic feature of this model:

$\psi_{(E)}=\frac{c}{2}\left(e^{Q}-1\right)$

$Q=b_{1} E_{\theta \theta}^{2}+b_{2} E_{Z Z}^{2}+b_{3} E_{R R}^{2}+2 b_{4} E_{\theta \theta} E_{Z Z}+2 b_{5} E_{Z Z} E_{R R}+2 b_{6} E_{R R} E_{\theta \theta}$

Where:

$\psi-$ is the strain energy density

$E_{i j}$ - are components of Green-Lagrange strain tensor $\mathrm{E}$ referred to cylindrical polar coordinates $(R, \Theta, Z)$ in the reference configuration

$\mathrm{C}-$ is the stress-like material parameter 
$b_{1}, b_{2} \ldots b_{3}$ are dimensions material parameters

\subsubsection{Polynomial (Aniso) Model}

Polynomial (Aniso) model is hyperelastic anisotropic material model defined through polynomial series of strain invariants $\left(\mathrm{l}_{\mathrm{i}}\right)$. The model definition is adopted from the ANSYS finite element package and simplified to symmetrical configuration of fibre families and to only $I_{1}, I_{2}, I_{4}\left(I_{6}\right)$ dependence. Incompressible formulation of this model is used in Hyperfit implementation.

$\psi_{\left(I_{1}, I_{2}, I_{4}, I_{6}\right)}=\sum_{i=1}^{3} a_{i}\left(I_{1}-3\right)^{i}+\sum_{j=1}^{3} b_{j}\left(I_{2}-3\right)^{j}+\sum_{k=2}^{6} c_{k}\left(I_{4}-1\right)^{k}+\sum_{m=2}^{6} e_{m}\left(I_{6}-1\right)^{m}$

$$
\begin{aligned}
I_{4}=C: A_{0} ; \quad I_{6}=C: B_{0} \\
A_{0}=a_{0} \otimes a_{0} ; \quad B_{0}=b_{0} \otimes b_{0}
\end{aligned}
$$

for the specific coordinate system (corresponding to the supported data protocols) and for the symmetric configuration of fibre families:

$a_{0}=\left[\begin{array}{c}\cos \varphi \\ \sin \varphi \\ 0\end{array}\right] ; \quad b_{0}=\left[\begin{array}{c}\cos \varphi \\ -\sin \varphi \\ 0\end{array}\right]$

Where:

$\Psi$ - is strain energy density

$I_{1}, I_{2}, I_{4}, I_{6}$ - are (pseudo-) invariants of $C$ (and $\left.A_{0}, B_{0}\right)$

$\mathrm{C}$ - is right Cauchy-Green def. tensor

$A_{0}, B_{0}$ - are structural tensors referenced to individual family of fibres

$a_{0}, b_{0}$ - are the direction vectors defining the orientation of fibre families

$a_{i}, b_{j}$ - are stress-like material parameters referenced to isotropic (matrix) properties

$c_{k}=\left(=e_{k}\right)-$ are stress-like material parameters referenced to anisotropic (fiber) properties

$\varphi$ - is material parameter defining the orientation angle of fibres (measured from axis "1") in the undeformed configuration.

\subsubsection{Holzapfel (2000) Model}


The Holzapfel (2000) model is a hyperelastic anisotropic material model proposed by Gerhard Holzapfel et al [29] for stress-strain description of arterial layers. The model is characterised by an isotropic matrix of neo Hookean form and anisotropic contribution of two symmetrical (and mechanically equivalent) families of (collagen) fibres reinforcing a vessel wall layer. Incompressible and Nearly-Incompressible (NI) formulations of this model are available in Hyperfit.

$\Psi_{\left(I_{1}, I_{4}, I_{6}\right)}=\Psi_{g\left(I_{1}\right)}+\Psi_{f\left(I_{4}, I_{6}\right)}$

$\Psi_{g\left(I_{1}\right)}=\frac{\mu}{2}\left(I_{1}-3\right)$

$\Psi_{g\left(I_{4}, I_{6}\right)}=\frac{k_{1}}{2 k_{2}} \sum_{i=4,6}\left(e^{k_{2}\left(I_{i}-1\right) 1^{2}}-1\right)$

Where:

$\Psi$ - is strain energy density

$I_{1}, I_{2}, I_{4}$ - are (pseudo-)invariants of $C$ (and $\left.A_{0}, B_{0}\right)$

is stress-like material parameter referenced to matrix properties

$\mathrm{k}_{1}$ - is stress-like material parameter referenced to fibre properties

$\mathrm{k}_{2}$ - is dimensionless material parameter referenced to fibre properties

$\varphi$ - is material parameter defining the orientation angle of fibres (measured from axis "1") in the undeformed configuration

$\mathrm{d}$ - is material parameter related to volumetric compressibility

\subsubsection{Holzapfel (2005) Model}

The Holzapfel (2005) model is a hyperelastic anisotropic material model proposed by Gerhard Holzapfel et al [18] for stress-strain description of arterial layers. The model represents a composite material reinforced by two families of (collagen) fibres that are mechanically equivalent and arranged in symmetrical spirals (in arterial layer). Incompressible formulation of this model is used in Hyperfit implementation.

$\Psi=\mu\left(I_{1}-3\right)+\sum_{i=1,2} \Psi_{f, i}\left(I_{1}, I_{4 i}\right)$ 
$\Psi_{f, i}=\frac{k_{1}}{2 k_{2}}\left(e^{k_{2}\left[(1-\rho)\left(I_{1}-3\right)^{2}+\rho\left(I_{4 i}-1\right)^{2}\right]}-1\right)$

$I_{4 i}=A_{0 i}: C$

$A_{0 i}=a_{0 i} \otimes a_{o i}$

$a_{01}=\left[\begin{array}{c}\cos \varphi \\ \sin \varphi \\ 0\end{array}\right] ; a_{02}=\left[\begin{array}{c}\cos \varphi \\ -\sin \varphi \\ 0\end{array}\right]$

$\Psi$ - is strain energy density

$I_{1}, I_{4}{ }_{1}, I_{4} 2$ - are (pseudo-)invariants of $C$

$\mathrm{C}$ - is right Cauchy-Green def. tensor

$A_{01}, A_{02}$ - are structural tensors referenced to individual family of fibres

$a_{01}, a_{02}$ - are the direction vectors defining the orientation of fibre families

$\mu$ - is stress-like material parameter referenced to matrix properties

$\mathrm{k}_{1}$ - is stress-like material parameter referenced to fibre properties

$\mathrm{k}_{2}$ - is dimensionless material parameter referenced to fibre properties

$\varphi$ - is structure parameter defining the (mean) orientation angle of fibre families (measured from axis "1") in the undeformed configuration

$\rho$ - is the dimensionless parameter that can be seen as a weighting factor (from 0 to

1: value of 0 specifies isotropic model similar to the Demiray model, value of 1 leads to Holzapfel, 2000 model)

\subsubsection{Four-fibre Family model (FFF)}

Four-fiber Family model is a hyperelastic anisotropic material model adopted from Ferruzzi et al [21] that refers to Baek et al [30]. The model is normally utilised for stressstrain description of aortas and aneurysms. The model represents an elastindominated amorphous matrix reinforced by four families of (collagen) fibres (in axial, circumferential and diagonal directions). The third constitutive model, called the Fourfibre Family (FFF), is a hyperelastic anisotropic model proposed by Baek et al [30] and Ferruzzi et al [21] for the stress-strain description of aortas and aneurysms. It is a variation of the HGO model, in which two more fibre families are added in longitudinal and circumferential orientations, respectively. This model therefore presents a total of 
four families of fibres; one axial, one circumferential and two symmetrical in diagonal directions. Its SEF adopts the following express:

$\Psi_{\left(C_{2} A_{0 i}\right)}=\Psi_{g(C)}+\Psi_{f\left(C_{2} A_{0 i}\right)}$

$\Psi_{g(C)}=\frac{1}{2}\left(I_{1}-3\right)$

$\Psi_{f\left(C, A_{0 i}\right)}=\sum_{i=1}^{4} \frac{c_{1 i}}{4 c_{2 i}}\left\{\exp \left[c_{2 i}\left(I_{4 i}-1\right)^{2}\right]-1\right\}$

Where:

$a_{0 i}=\left[\begin{array}{l}0 \\ 1 \\ 0\end{array}\right] ; a_{02}=\left[\begin{array}{l}1 \\ 0 \\ 0\end{array}\right] ; a_{03}=\left[\begin{array}{c}\cos \varphi_{0} \\ \sin \varphi_{0} \\ 0\end{array}\right] ; a_{04}=\left[\begin{array}{c}\cos \varphi_{0} \\ -\sin \varphi_{0} \\ 0\end{array}\right]$

$\Psi$ is strain energy density

$I_{1}$ is the first invariant of $C$

$\mathrm{I}_{4 \mathrm{i}}$ are (pseudo-)invariants of $\mathrm{C}$ and $\mathrm{AO} \mathrm{i}(\mathrm{i}=1$ to 4$)$

$\mathrm{C}$ is right Cauchy-Green def. tensor

$A_{01}, A_{02}$ are structural tensors referenced to individual fibre families ( $i=1$ to 4 )

$a_{01}, a_{02}$ are unit vectors defining the orientation of individual fibre families ( $i=1$ to $4, i=1$

axis 2 (axial), $\mathrm{i}=2 \sim$ axis 1 (circ.), $\mathrm{i}=3,4 \sim$ diagonal dirs.)

$\mathrm{C}$ is stress-like material parameter referenced to matrix properties

$\mathrm{C}_{1 \mathrm{i}}(\mathrm{i}=1,2,34)$ are stress-like material parameters referenced to properties of individual fibre families $c_{2 i}(i=1,2,34)$ are dimensionless material parameters referenced to properties of individual fibre families

$\varphi_{0}$ is structure parameter defining the orientation angle of diagonal fibre families (measured from axis "1") in the undeformed configuration

\subsection{Numerical analysis}

This algorithm is the COBYLA method implemented as third-party library. SciPy library is adopted for this implementation. COBYLA method performs constrained optimisation by the linear approximation method. Coefficient of determination $\left(R^{2}\right)$ (also known as Nash-Sutcliffe coefficient) 
$R^{2}=1-\frac{\sum_{i=1}^{n}\left(y_{e}-y_{m}\right)^{2}}{\sum_{i=1}^{n}\left(y_{e}-\overline{y_{e}}\right)^{2}}$

(24)

Range:

$R^{2} \epsilon\langle-\infty, 1\rangle$

For perfect fit:

$R^{2}=1$

Where:

$\mathrm{y}_{\mathrm{e}}$ is experimental (observed) value of the fitted function

$y_{m}$ is the model (theoretical) value of the fitted function

$i$ is the data point index

$\mathrm{n}$ is the number of data-points

$\mathrm{y}_{\mathrm{e}}$ is the mean value from the experimental values calculated as follows:

$\overline{y_{e}}=\frac{1}{n} \sum_{i=1}^{n} y_{e}$

(25)

Correlation coefficient ( $r$ ) is defined as follows:

$r=\frac{\sum_{i=1}^{n}\left(y_{e}-\overline{y_{e}}\right)\left(y_{m}-\overline{y_{m}}\right)}{\sqrt{\sum_{i=1}^{n}\left(y_{m}-\overline{y_{m}}\right)^{2}} \cdot \sum_{i=1}^{n}\left(y_{m}-\overline{y_{m}}\right)^{2}}$

(26)

Where:

$\overline{y_{m}}$ is the mean value from the models values (calculated below):

$\overline{y_{m}}=\frac{1}{n} \sum_{i=1}^{n} y_{m}$

Normalized RMS error (NRMSE) is defined as follows:

$N R M S E=\frac{\sqrt{\frac{1}{n}} \sum_{i=1}^{n}\left(y_{e}-y_{m}\right)^{2}}{a b s\left(\overline{y_{e}}\right)}$

(30)

Normalised error (NE) is defined as follows:

$N E=\frac{\frac{1}{n} \sum_{i=1}^{n} a b s\left(y_{e}-y_{m}\right)}{a b s\left(\overline{y_{e}}\right)}$ 


\section{Results}

Figure 2 shows the stress-strain curves for all the samples (Test 1-12) tested on the sheep Omasum. The stresses are plotted up to $50 \%$ strain, although half of the samples were tested up to $35 \%$ strain. Some of the stress-strain curves exhibit long toe regions that extend up to $25 \%$ strain.

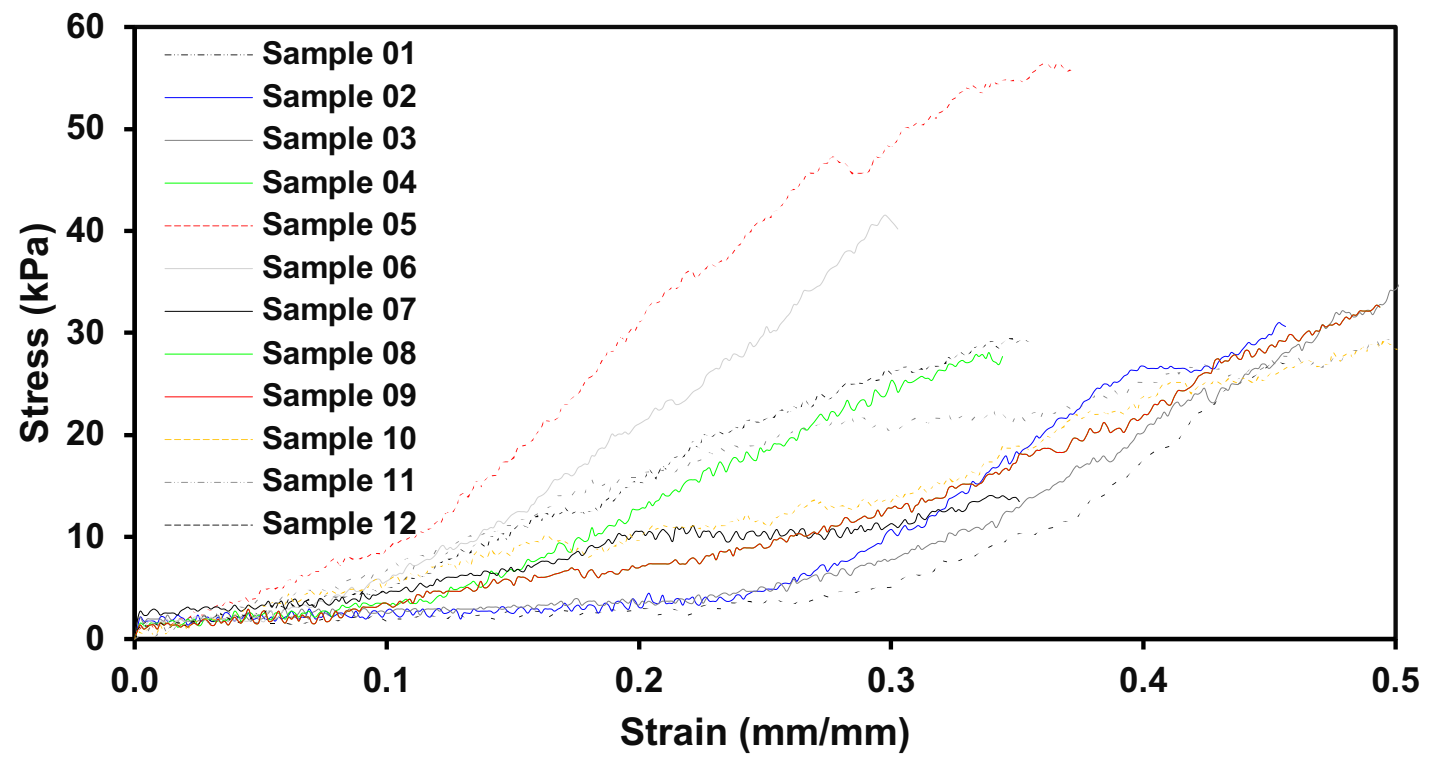

Figure 2. Stress vs Strain of the sheep Omasum for all the tested samples

The elastic modulus was calculated two strain levels (15 and 20\%). Figure 3 shows the elastic modulus of sheep omasum under axial loading for all the samples. Sample 5 and 6 shows the highest elastic modulus at both the $15 \%$ and $20 \%$ strain.

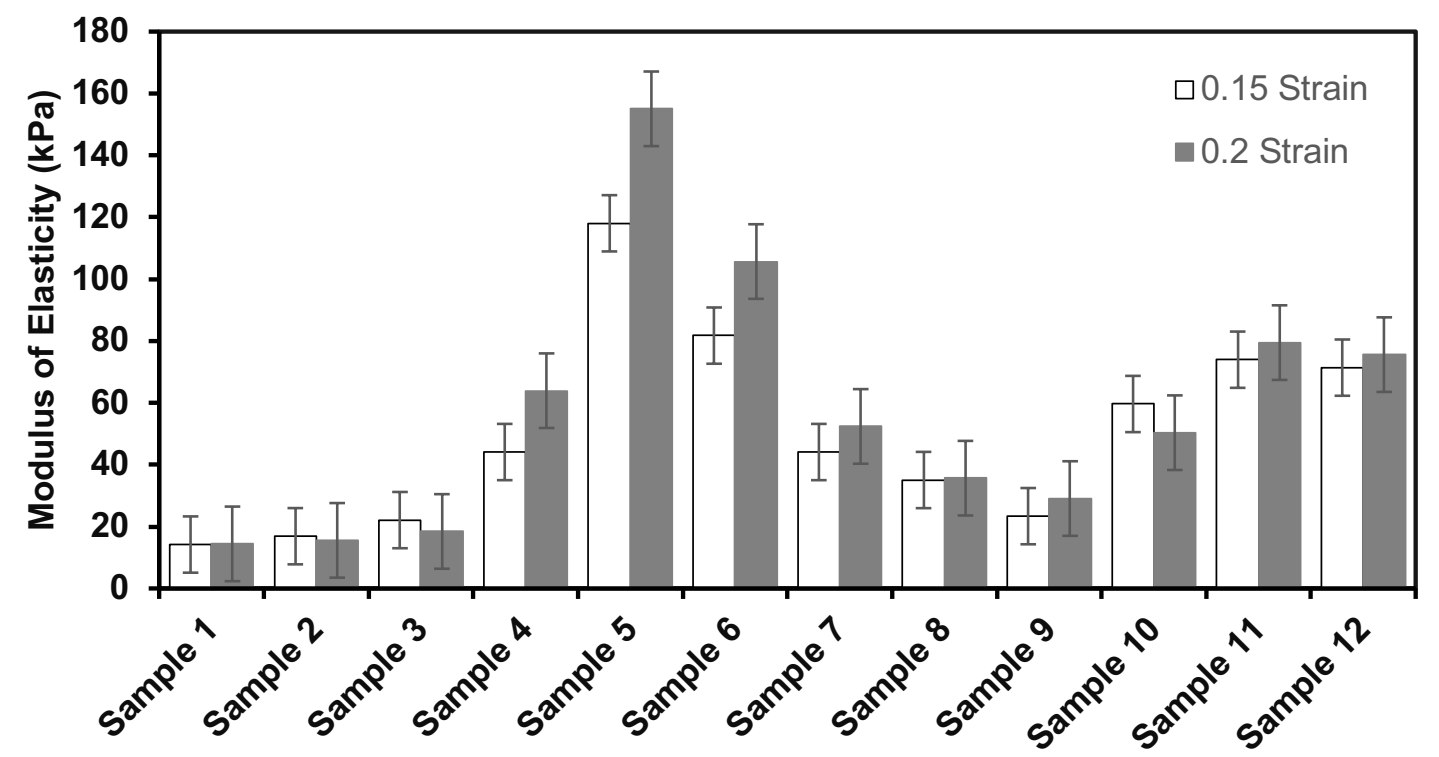

Figure 3. Elastic modulus of sheep omasum under axial loading up to $15 \%$ and $20 \%$ strain 
To examine the exact trend of the stress-strain curves of all the samples relative to each other, the stress was calculated at $30 \%$ strain as it covered all the samples and plotted in Figure 4.

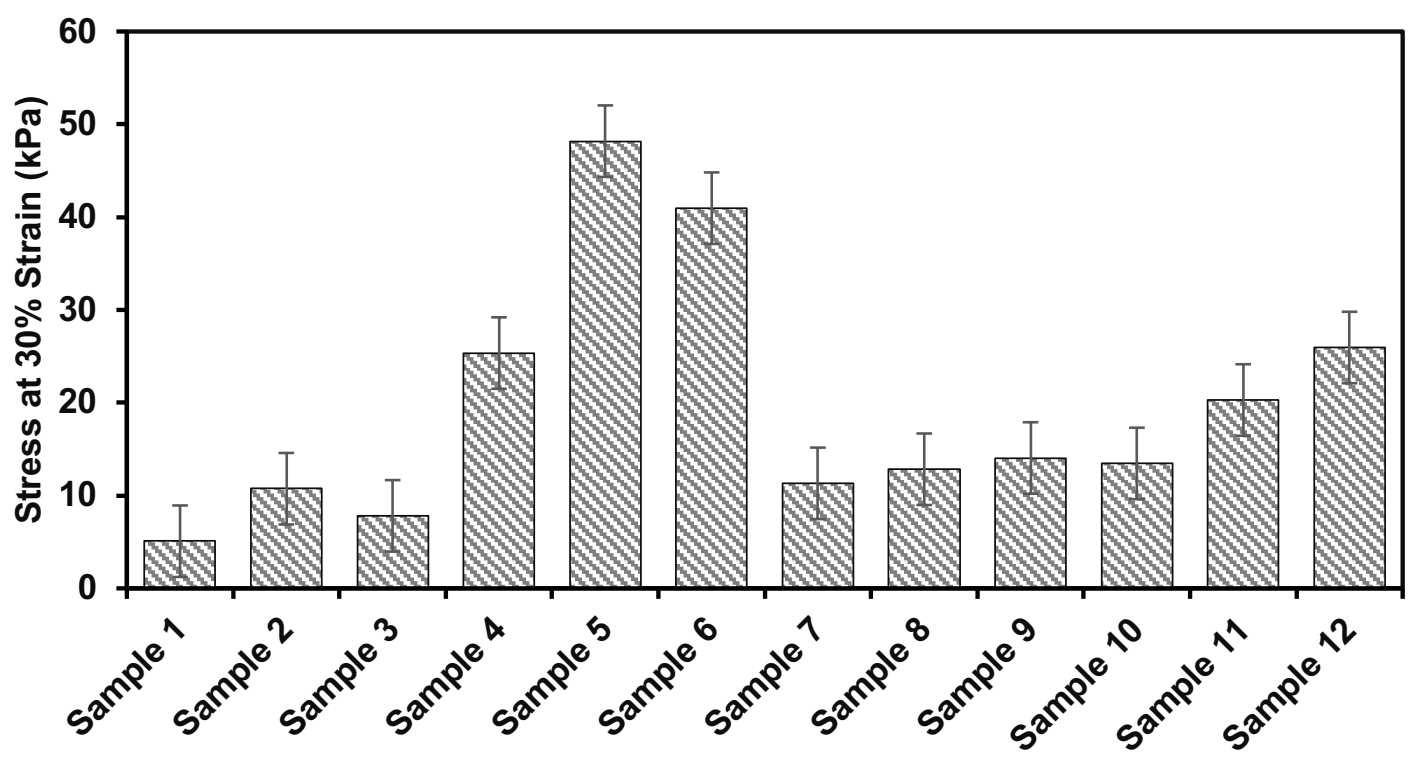

Figure 4. Stress value of all the samples at $30 \%$ of strain

The study investigated material parameters differences across the sheep Omasum samples using five hyperelastic different models and their prediction capabilities. The models were the Fung, the Polynomial model, the two Holzapfel (2000 and 2005) and four-fiber-family models. The model material properties are presented for the five models in Table 1 to Table 5.

Table 1. Fung hyperelastic model material parameters identification from uniaxial test of the sheep omasum

\begin{tabular}{|c|c|c|c|c|c|c|c|c|c|c|c|}
\hline $\begin{array}{c}\text { Sampl } \\
\mathrm{e}\end{array}$ & $\mathrm{c}$ & $\mathrm{b}_{1}$ & $\mathrm{~b}_{2}$ & $\mathrm{~b}_{3}$ & $\mathrm{~b}_{4}$ & $\mathrm{~b}_{5}$ & $\mathrm{~b}_{6}$ & $\mathrm{R}^{2}$ & $\mathbf{r}$ & $\begin{array}{c}\text { NRMS } \\
\mathrm{E}\end{array}$ & $\mathbf{N E}$ \\
\hline 1 & 3.57 & 3.29 & 0.46 & -0.14 & 0.01 & 0.15 & -0.02 & 0.96 & 0.98 & 0.20 & 0.17 \\
\hline 2 & 3.61 & 3.81 & 3.54 & 0.27 & 0.27 & 0.25 & 0.00 & 0.94 & 0.97 & 0.23 & 0.18 \\
\hline 3 & 3.59 & 2.87 & 2.59 & 0.23 & 0.21 & 0.18 & 0.00 & 0.93 & 0.98 & 0.26 & 0.20 \\
\hline 4 & 3.64 & 6.51 & 1.36 & 0.59 & -0.75 & -0.18 & -0.06 & 0.89 & 0.96 & 0.25 & 0.19 \\
\hline 5 & 3.59 & 8.23 & 2.59 & 0.45 & -0.78 & -0.16 & -0.05 & 0.49 & 0.87 & 0.49 & 0.41 \\
\hline 6 & 3.65 & 9.70 & 1.28 & 0.61 & -0.82 & -0.17 & -0.07 & 0.92 & 0.97 & 0.23 & 0.18 \\
\hline 7 & 3.65 & 4.33 & 1.35 & 1.11 & -1.05 & -0.16 & -0.15 & 0.44 & 0.93 & 0.35 & 0.32 \\
\hline 8 & 3.65 & 3.42 & 1.50 & 0.69 & -0.59 & -0.18 & -0.05 & 0.88 & 0.98 & 0.26 & 0.23 \\
\hline 9 & 3.59 & 2.80 & 2.59 & 0.29 & 0.31 & 0.27 & 0.00 & 0.71 & 0.92 & 0.42 & 0.34 \\
\hline 10 & 3.69 & 3.16 & 1.45 & 1.09 & -0.73 & -0.19 & -0.08 & 0.61 & 0.94 & 0.38 & 0.35 \\
\hline 11 & 3.68 & 6.44 & 1.27 & 1.05 & -0.97 & -0.18 & -0.13 & 0.75 & 0.93 & 0.93 & 0.28 \\
\hline 12 & 5.34 & 1.19 & 0.30 & -1.89 & -0.03 & -0.22 & 0.12 & 0.92 & 0.96 & 0.29 & 0.26 \\
\hline Mean & 3.77 & 4.65 & 1.69 & 0.36 & -0.41 & -0.05 & -0.04 & 0.79 & 0.95 & 0.36 & 0.26 \\
\hline SD & 0.50 & 2.52 & 0.95 & 0.81 & 0.52 & 0.19 & 0.07 & 0.19 & 0.03 & 0.20 & 0.08 \\
\hline
\end{tabular}


Table 2. Polynomial (anisotropic) hyperelastic model material parameters $\left(\mathbf{a}_{1}, \mathbf{a}_{2}\right.$, $a_{3}, b_{1}, b_{2}, b_{3}, c_{2}, c_{3}, c_{5}, c_{6}, \varphi$ and $R^{2}$ ) identification from uniaxial test of the sheep omasum

\begin{tabular}{|c|c|c|c|c|c|c|c|c|c|c|c|c|c|c|c|c|}
\hline \begin{tabular}{|l|} 
Sampl \\
$e^{-2}$
\end{tabular} & $a_{1}$ & $a_{2}$ & $a_{3}$ & $b_{1}$ & $b_{2}$ & $b_{3}$ & $\mathrm{C}_{2}$ & $\mathrm{C}_{3}$ & $\mathrm{C}_{4}$ & $\mathrm{C}_{5}$ & $\mathrm{C}_{6}$ & $\varphi$ & $\mathrm{R}^{2}$ & $r$ & $\begin{array}{c}\text { NRM } \\
\text { SE }\end{array}$ & $\mathrm{NE}$ \\
\hline 1 & 2.90 & 4.85 & 4.88 & $\begin{array}{c}10.8 \\
5\end{array}$ & 10.85 & 10.85 & -0.65 & 0.21 & 0.12 & 0.07 & 0.04 & $\begin{array}{c}- \\
0.06\end{array}$ & $\begin{array}{l}0.9 \\
8\end{array}$ & $\begin{array}{c}0.9 \\
9\end{array}$ & 0.16 & 0.14 \\
\hline 2 & 4.95 & 7.23 & 4.65 & $\begin{array}{c}11.7 \\
4\end{array}$ & 11.74 & \begin{tabular}{|l|l|}
11.74 \\
\end{tabular} & -0.77 & 0.30 & 0.13 & 0.05 & 0.02 & 0.00 & $\begin{array}{l}0.9 \\
6\end{array}$ & $\begin{array}{c}0.9 \\
8\end{array}$ & 0.19 & 0.16 \\
\hline 3 & 13.35 & 2.36 & 1.07 & 2.59 & 2.59 & 2.59 & $\mid-0.34$ & 0.10 & 0.03 & 0.01 & 0.00 & 0.00 & $\begin{array}{c}0.8 \\
0\end{array}$ & $\begin{array}{c}0.9 \\
6\end{array}$ & 0.45 & 0.38 \\
\hline 4 & 13.32 & 5.70 & 4.59 & 5.18 & 5.18 & 5.18 & -0.41 & 0.23 & 0.12 & 0.06 & 0.03 & 0.00 & $\begin{array}{l}0.9 \\
5\end{array}$ & $\begin{array}{c}0.9 \\
9\end{array}$ & 0.18 & 0.14 \\
\hline 5 & 32.04 & 3.35 & 1.30 & 2.59 & 2.59 & 2.59 & -0.24 & 0.14 & 0.04 & 0.01 & 0.00 & 0.00 & $\begin{array}{l}0.9 \\
4\end{array}$ & $\begin{array}{c}0.9 \\
9\end{array}$ & 0.16 & 0.12 \\
\hline 6 & 19.97 & $\begin{array}{c}11.7 \\
5\end{array}$ & $\begin{array}{c}14.6 \\
5\end{array}$ & 5.18 & 5.18 & 5.18 & -0.78 & 0.47 & 0.31 & 0.22 & 0.17 & 0.00 & $\begin{array}{l}0.9 \\
6\end{array}$ & $\begin{array}{c}0.9 \\
9\end{array}$ & 0.16 & 0.13 \\
\hline 7 & 8.84 & -0.61 & -0.33 & 5.24 & 5.24 & 5.24 & 0.04 & -0.03 & -0.01 & 0.00 & 0.00 & 0.00 & $\begin{array}{l}0.9 \\
4\end{array}$ & $\begin{array}{c}0.9 \\
8\end{array}$ & 0.11 & 0.09 \\
\hline 8 & 10.08 & 2.58 & 1.61 & 5.18 & 5.18 & 5.18 & -0.31 & 0.11 & 0.05 & 0.02 & 0.01 & 0.00 & $\begin{array}{l}0.9 \\
6\end{array}$ & $\begin{array}{c}0.9 \\
9\end{array}$ & 0.16 & 0.13 \\
\hline 9 & 11.55 & 2.14 & 0.70 & 5.18 & 5.18 & 5.18 & -0.26 & 0.08 & 0.02 & 0.01 & 0.00 & 0.00 & $\begin{array}{l}0.9 \\
4\end{array}$ & $\begin{array}{c}0.9 \\
8\end{array}$ & 0.20 & 0.14 \\
\hline 10 & 11.46 & 1.17 & 0.65 & 5.18 & 5.18 & 5.18 & -0.14 & 0.05 & 0.02 & 0.01 & 0.00 & 0.00 & $\begin{array}{l}0.9 \\
8\end{array}$ & $\begin{array}{c}0.9 \\
9\end{array}$ & 0.10 & 0.07 \\
\hline 11 & 14.62 & -0.44 & -0.26 & 5.25 & 5.25 & 5.25 & 0.06 & -0.02 & -0.01 & 0.00 & 0.00 & 0.00 & $\begin{array}{l}0.9 \\
9\end{array}$ & $\begin{array}{c}0.9 \\
9\end{array}$ & 0.06 & 0.05 \\
\hline 12 & 3.48 & 5.82 & 5.85 & $\begin{array}{c}10.8 \\
5\end{array}$ & 10.85 & 10.85 & -0.78 & 0.25 & 0.15 & 0.09 & 0.05 & $\begin{array}{c}- \\
0.06\end{array}$ & $\begin{array}{l}0.9 \\
8\end{array}$ & $\begin{array}{c}0.9 \\
9\end{array}$ & 0.16 & 0.14 \\
\hline Mean & 12.21 & 3.83 & 3.28 & 6.25 & 6.25 & 6.25 & -0.38 & 0.16 & 0.08 & 0.05 & 0.03 & $\begin{array}{c}- \\
0.01\end{array}$ & $\begin{array}{l}0.9 \\
5\end{array}$ & $\begin{array}{c}0.9 \\
9\end{array}$ & 0.17 & 0.14 \\
\hline SD & 7.94 & 3.50 & 4.18 & 3.12 & 3.12 & 3.12 & 0.30 & 0.14 & 0.09 & 0.06 & 0.05 & 0.02 & 0.05 & \begin{tabular}{|l|l}
0.01 \\
\end{tabular} & 0.10 & 0.08 \\
\hline
\end{tabular}

Table 3: Holzapfel (2000) hyperelastic model material parameters $\left(\mu, \mathrm{k}_{1}, \mathrm{k}_{2}, \varphi\right.$, and $R^{2}$ ) identification from uniaxial test of the sheep omasum

\begin{tabular}{|l|c|c|c|c|c|c|c|c|}
\hline Sample & $\mu$ & $\mathrm{k}_{1}$ & $\mathrm{k}_{2}$ & $\varphi$ & $\mathrm{R}^{2}$ & $\mathrm{r}$ & $\mathrm{NRMSE}$ & $\mathrm{NE}$ \\
\hline 1 & 3.54 & 9.73 & 48.94 & 0.79 & 0.98 & 0.99 & 0.15 & 0.12 \\
\hline 2 & 16.45 & 7.07 & 13.10 & 0.81 & 0.81 & 0.94 & 0.42 & 0.35 \\
\hline 3 & 25.52 & 4.44 & 3.88 & 0.80 & 0.76 & 0.94 & 0.49 & 0.42 \\
\hline 4 & 26.54 & 6.25 & 24.06 & 0.81 & 0.91 & 0.98 & 0.23 & 0.18 \\
\hline 5 & 63.33 & 4.04 & 8.48 & 0.81 & 0.94 & 0.99 & 0.17 & 0.13 \\
\hline 6 & 40.08 & 10.06 & 44.00 & 0.81 & 0.92 & 0.98 & 0.23 & 0.19 \\
\hline 7 & 16.80 & 0.42 & 0.00 & 0.73 & 0.93 & 0.98 & 0.12 & 0.09 \\
\hline 8 & 19.95 & 4.62 & 8.10 & 0.81 & 0.92 & 0.98 & 0.21 & 0.19 \\
\hline 9 & 22.68 & 4.26 & 3.87 & 0.81 & 0.91 & 0.98 & 0.23 & 0.18 \\
\hline 10 & 22.43 & 2.54 & 5.01 & 0.81 & 0.97 & 0.99 & 0.11 & 0.09 \\
\hline 11 & 27.76 & 0.38 & 0.00 & 0.69 & 0.98 & 0.99 & 0.07 & 0.06 \\
\hline 12 & 4.25 & 11.31 & 48.65 & 0.79 & 0.98 & 0.99 & 0.15 & 0.12 \\
\hline Mean & 24.11 & 5.43 & 17.34 & 0.79 & 0.92 & 0.98 & 0.22 & 0.18 \\
\hline SD & 15.86 & 3.59 & 19.15 & 0.04 & 0.07 & 0.02 & 0.12 & 0.11 \\
\hline
\end{tabular}


Table 4: Holzapfel (2005) hyperelastic model material parameters $\left(\mu, \mathrm{k}_{1}, \mathrm{k}_{2}\right.$, $\varphi, \rho$, and $\mathrm{R}^{2}$ ) identification from uniaxial test of the sheep omasum

\begin{tabular}{|c|c|c|c|c|c|c|c|c|c|}
\hline Sample & $\mu$ & $\mathrm{k}_{1}$ & $\mathrm{k}_{2}$ & $\varphi$ & $\rho$ & $\mathrm{R}^{2}$ & $\mathrm{r}$ & $\mathrm{NRMSE}$ & $\mathrm{NE}$ \\
\hline 1 & 1.89 & 6.96 & 6.75 & -0.95 & 0.37 & 0.98 & 0.99 & 0.14 & 0.12 \\
\hline 2 & 7.69 & 5.64 & 4.38 & -0.90 & 0.35 & 0.86 & 0.95 & 0.36 & 0.30 \\
\hline 3 & 12.42 & 3.37 & 1.89 & -0.85 & 0.43 & 0.80 & 0.96 & 0.45 & 0.38 \\
\hline 4 & 12.94 & 6.13 & 8.94 & -0.90 & 0.21 & 0.93 & 0.98 & 0.21 & 0.16 \\
\hline 5 & 31.66 & 4.08 & 3.85 & -0.88 & 0.23 & 0.94 & 0.99 & 0.17 & 0.13 \\
\hline 6 & 19.45 & 10.73 & 18.03 & -0.91 & 0.14 & 0.94 & 0.99 & 0.19 & 0.15 \\
\hline 7 & 8.27 & 0.44 & 0.00 & 0.63 & 1.00 & 0.93 & 0.98 & 0.12 & 0.09 \\
\hline 8 & 9.57 & 3.68 & 3.31 & -0.89 & 0.37 & 0.95 & 0.99 & 0.18 & 0.15 \\
\hline 9 & 10.92 & 2.93 & 1.65 & 0.87 & 0.39 & 0.93 & 0.98 & 0.20 & 0.15 \\
\hline 10 & 10.97 & 2.04 & 2.16 & 0.88 & 0.37 & 0.97 & 0.99 & 0.10 & 0.08 \\
\hline 11 & 13.52 & 0.45 & -0.01 & 0.52 & 1.00 & 0.98 & 0.99 & 0.08 & 0.06 \\
\hline 12 & 2.30 & 8.17 & 6.82 & -0.95 & 0.37 & 0.98 & 0.99 & 0.14 & 0.12 \\
\hline Mean & 11.80 & 4.55 & 4.81 & -0.36 & 0.44 & 0.93 & 0.98 & 0.19 & 0.16 \\
\hline SD & 7.87 & 3.10 & 5.00 & 0.81 & 0.28 & 0.05 & 0.01 & 0.11 & 0.09 \\
\hline
\end{tabular}

Table 5: Four-Fiber-Family hyperelastic model material parameters $\left(c, c_{11}, c_{21}\right.$, , $\mathrm{C}_{21}, \mathrm{C}_{12}, \mathrm{C}_{22}, \mathrm{C}_{134}, \mathrm{C}_{234}, \varphi_{0}$, and $\mathrm{R}^{2}$ ) identification from uniaxial test of the sheep omasum

\begin{tabular}{|l|c|c|c|c|c|c|c|c|l|l|l|l|}
\hline Sample & $\mathrm{c}$ & $\mathrm{C}_{11}$ & $\mathrm{C}_{21}$ & $\mathrm{C}_{12}$ & $\mathrm{C}_{22}$ & $\mathrm{C}_{134}$ & $\mathrm{C}_{234}$ & $\varphi_{0}$ & $\mathrm{R}^{2}$ & $\mathrm{r}$ & $\mathrm{NRMSE}$ & $\mathrm{NE}$ \\
\hline 1 & 2.16 & 21.09 & 371605.07 & 2.70 & 1.49 & 11707.67 & 3.74 & 1.13 & 0.98 & 0.99 & 0.14 & 0.11 \\
\hline 2 & 13.75 & 0.74 & 0.00 & 2.45 & 1.28 & -0.06 & 0.27 & 0.00 & 0.87 & 0.96 & 0.35 & 0.29 \\
\hline 3 & 18.72 & 1.32 & 9497.88 & 1.34 & 1.01 & -0.01 & 0.00 & 0.00 & 0.92 & 0.98 & 0.29 & 0.24 \\
\hline 4 & 24.86 & 1.92 & 32045.40 & 2.48 & 2.00 & 0.00 & 163.06 & 0.78 & 0.92 & 0.98 & 0.21 & 0.17 \\
\hline 5 & 61.81 & 1.32 & 0.00 & 1.88 & 1.41 & 0.00 & 27.28 & 0.79 & 0.94 & 0.99 & 0.17 & 0.13 \\
\hline 6 & 37.98 & 0.72 & 0.00 & 3.60 & 3.14 & 11.19 & 0.00 & 2.12 & 0.93 & 0.99 & 0.21 & 0.17 \\
\hline 7 & 15.36 & 15.49 & 55032.35 & 0.65 & 0.00 & 2.69 & 0.00 & 0.75 & 0.92 & 0.98 & 0.13 & 0.10 \\
\hline 8 & 17.33 & 1.34 & 1487.15 & 1.67 & 1.11 & 0.00 & 58.24 & 1.68 & 0.96 & 0.99 & 0.15 & 0.13 \\
\hline 9 & 18.28 & 1.16 & 523965.65 & 1.02 & 0.97 & 3.16 & 0.27 & 0.78 & 0.94 & 0.98 & 0.18 & 0.16 \\
\hline 10 & 19.60 & 1.29 & 5523.90 & 1.02 & 0.98 & 1.55 & 0.93 & 0.79 & 0.98 & 0.99 & 0.09 & 0.07 \\
\hline 11 & 24.45 & 1.81 & 35573.78 & 0.34 & 0.54 & 4.90 & -0.01 & 0.72 & 0.96 & 0.98 & 0.10 & 0.08 \\
\hline 12 & 2.71 & 2.26 & -0.01 & 3.28 & 1.47 & 0.03 & 33249.22 & 1.72 & 0.98 & 0.99 & 0.14 & 0.11 \\
\hline Mean & 21.42 & 4.20 & 86227.60 & 1.87 & 1.28 & 977.59 & 2791.92 & 0.94 & 0.94 & 0.98 & 0.18 & 0.15 \\
\hline SD & 15.90 & 6.70 & 172903.51 & 1.04 & 0.77 & 3379.10 & 9591.68 & 0.64 & 0.03 & 0.01 & 0.08 & 0.07 \\
\hline
\end{tabular}


Figure 5 shows the Evaluation Index (EI) with standard error based on coefficient of determination $\left(R^{2}\right)$ of individual samples for Fung, Polynomial (Anisotropic), Holzapfel (2000), Holzapfel (2005) and Four-Fiber-Family hyperelastic models showing the best model per sample based on the uniaxial testing of sheep omasum. The Polynomial (Anisotropic) model approximate the sheep Omasum behaviour better than the other models.

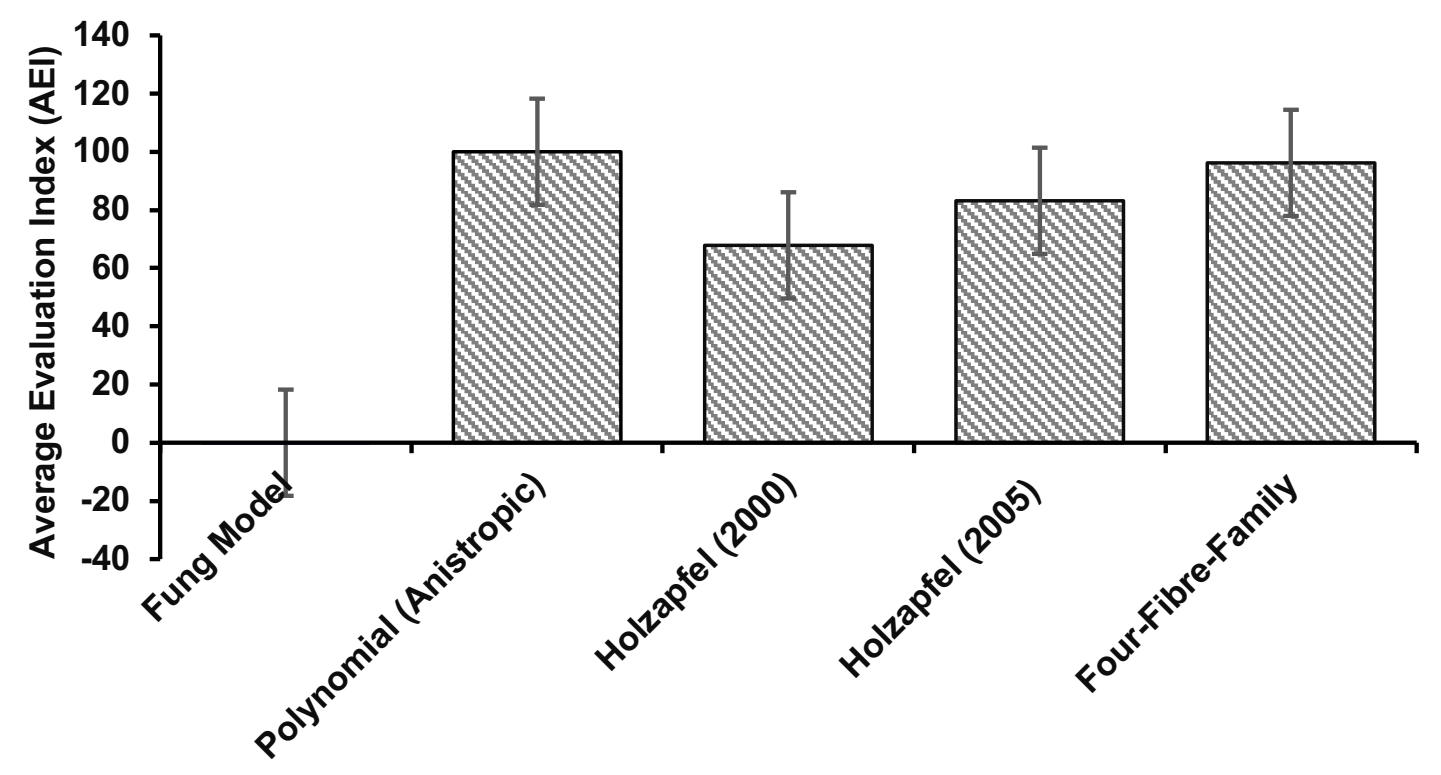

Figure 5: Evaluation Index (EI) with standard error based on average coefficient of determination $\left(\mathbf{R}^{2}\right)$ of Fung, Polynomial (Anisotropic), Holzapfel (2000), Holzapfel (2005) and Four-Fiber-Family hyperelastic models showing the best model based on the uniaxial testing of sheep omasum

To examine the exact differences between the five models, Normalised Error (NE) and Normalised RMS Error (NRMSE) were calculated and plotted in Figure 6. The NE and NRMSE results show that the errors from Fung model has the biggest error as compared to the other models.

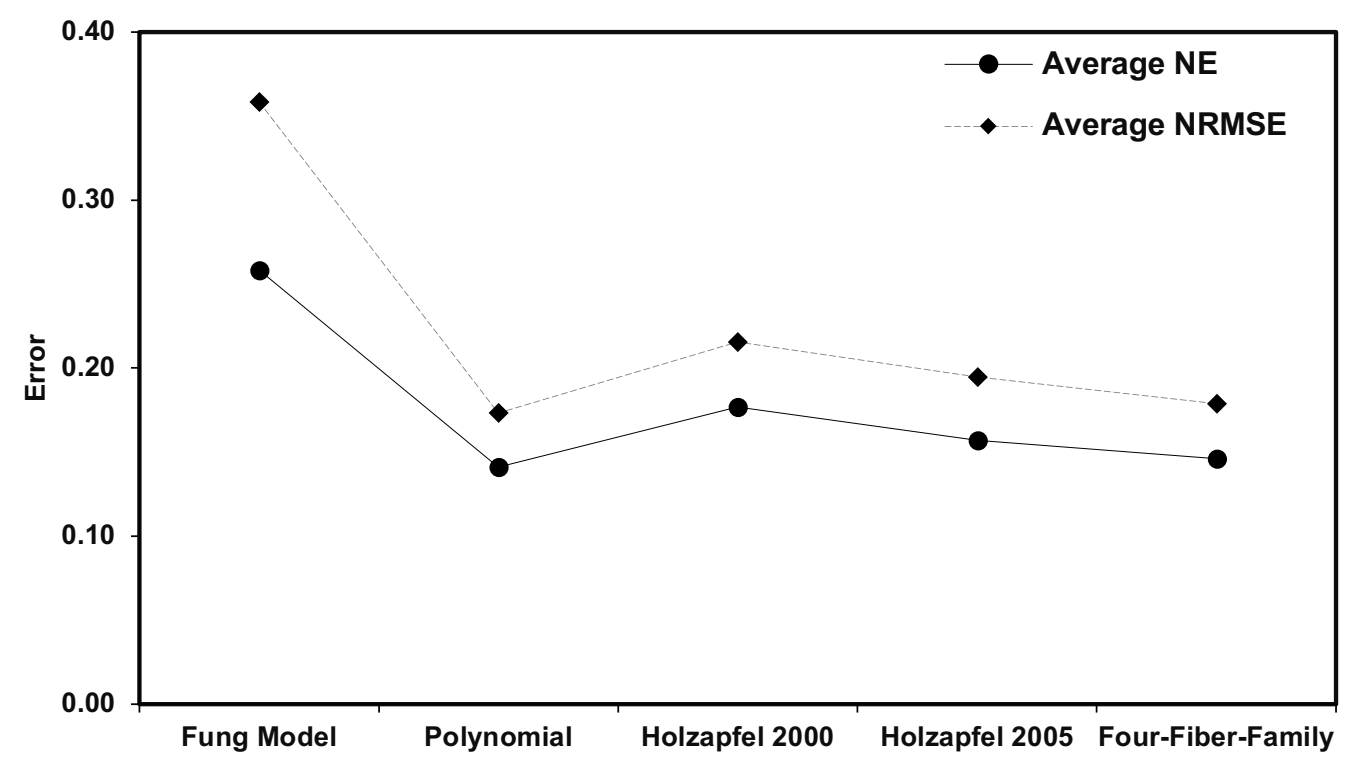




\section{Figure 6: Normalised Error (NE) and Normalised RMS Error (NRMSE) of Fung, Polynomial (Anisotropic), Holzapfel (2000), Holzapfel (2005) and Four-Fiber- Family hyperelastic models showing the best model based on the uniaxial testing of sheep omasum}

\section{Discussion}

This is the first study to investigate the mechanical materials properties of sheep Omasum using different hyperelastic models. The sheep omasum samples showed large variability in stress strain response during uniaxial testing (Figure 2 ). The large variability could be a result from variations in the regions of the Omasum where the samples were cut.

The results of the uniaxial tension tests of sheep Omasum stress-strain response were utilised to fit in the strain energy functions of Fung, Polynomial (Anisotropic), Holzapfel (2005), Holzapfel (2000) and Four-fiber family model. A direct comparison of hyperelastic constitutive models was made based on correlation coefficient $\left(R^{2}\right)$ and evaluation index (EI). The Polynomial (anisotropic) hyperelastic constitutive model was found to have the El of 100 . This means that it is the best performance constitutive model when compared to other four hyperelastic model considered in this study. In addition, Four-Fibre family hyperelastic constitutive model was found to have the El of 96 and therefore regarded as second best in terms of performances when fitted on the uniaxial experimental data of the sheep Omasum. This was followed by both the Holzapfel models $(2005,2000)$ with El of 83 and 63, respectively. Finally, the worst performer with EI of 0 was found to be Fung model.

\section{Limitations}

The measurement of sample thickness which was made difficult by the variable thickness of the Omasum. Errors in estimating the true sample thickness would affect the cross-sectional area, and hence the calculated stress.

\section{Conclusion}

As a first attempt to describe the mechanical material properties of the sheep omasum, five different hyperelatic models were used. This is the first step in understanding the mechanical material properties of Omasum and full characterisation of the tissue. The established anisotropic materials properties could be utilised in the understanding of tumours of the omasum. The identified material parameters will provide new platform for FE investigations of mechanical aspects of various therapies when using sheep omasum models. 


\section{Author contributions}

F.N, H.N and T.P contributed to conception, design, data acquisition, analysis, and interpretation, drafted the manuscript, L.L, T.P and F.N contributed to the interpretation of the data and critically revised the manuscript, and finally L.L, F.N, H.N and T.P contributed to conception, design, data analysis, and interpretation, drafted and critically revised the manuscript. All authors gave final approval and agree to be accountable for all aspects of the work.

\section{Competing interests}

The authors declare no competing interests.

Additional information

Correspondence and requests for materials should be addressed to F.N.

Reprints and permissions information is available at www.nature.com/reprints.

\section{Acknowledgments}

Support from the National Research Foundation (NRF) Grant number (129380) is gratefully acknowledged. Unisa CAPEX Programme supported the acquisition of biaxial testing machine in the Department of Mechanical Engineering, School of Engineering, College of Science Engineering and Technology.

\section{Corresponding author}

Correspondence to Fulufhelo Nemavhola

\section{References}

1. Grünberg, W. and P.D. Constable, Function and dysfunction of the ruminant forestomach, in Current Veterinary Therapy. 2009, Elsevier Inc. p. 12-19.

2. Movassaghi, A.R., G. Mohammadi, and M.R. Oghazi, Fibromyxoma in the reticulo-omasal orifice of a cow. Comparative Clinical Pathology, 2013. 22(3): p. 535-537.

3. Jiang, M., et al., A versatile biaxial testing platform for soft tissues. Journal of the Mechanical Behavior of Biomedical Materials, 2021. 114: p. 104144.

4. Egorov, V.I., et al., Mechanical properties of the human gastrointestinal tract. Journal of biomechanics, 2002. 35(10): p. 1417-1425.

5. Carniel, E.L., et al., Biomechanics of stomach tissues and structure in patients with obesity. Journal of the Mechanical Behavior of Biomedical Materials, 2020. 110: p. 103883.

6. Fontanella, C.G., et al., Computational models for the mechanical investigation of stomach tissues and structure. Annals of biomedical engineering, 2019. 47(5): p. 1237-1249.

7. Masithulela, F.J., Computational biomechanics in the remodelling rat heart post myocardial infarction. 2016.

8. Masithulela, F., Bi-ventricular finite element model of right ventricle overload in the healthy rat heart. Bio-medical materials and engineering, 2016. 27(5): p. 507-525.

9. Masithulela, F. The effect of over-loaded right ventricle during passive filling in rat heart: $A$ biventricular finite element model. in ASME International Mechanical Engineering Congress and Exposition. 2015. American Society of Mechanical Engineers.

10. Masithulela, F. Analysis of passive filling with fibrotic myocardial infarction. in ASME international mechanical engineering congress and exposition. 2015. American Society of Mechanical Engineers. 
11. Masithulela, F. The Effect of Over-Loaded Right Ventricle During Passive Filling in Rat Heart: A Biventricular Finite Element Model. in ASME 2015 International Mechanical Engineering Congress and Exposition. 2015.

12. Ndlovu, Z., F. Nemavhola, and D. Desai, BIAXIAL MECHANICAL CHARACTERIZATION AND CONSTITUTIVE MODELLING OF SHEEP SCLERA SOFT TISSUE. Russian Journal of Biomechanics/Rossijski Zurnal Biomehaniki, 2020. 24(1).

13. Nemavhola, F., Study of biaxial mechanical properties of the passive pig heart: material characterisation and categorisation of regional differences. International Journal of Mechanical and Materials Engineering, 2021. 16(1): p. 1-14.

14. Nemavhola, F., Biaxial quantification of passive porcine myocardium elastic properties by region. Engineering Solid Mechanics, 2017. 5(3): p. 155-166.

15. Nemavhola, F., et al., Passive Biaxial Tensile Dataset of Three Main Rat Heart Myocardia: Left Ventricle, Mid-Wall and Right Ventricle. Preprints, 2021. 2021080153(Version 1).

16. Ngwangwa, H., et al., Determination of Cross-directional and Cross-Wall Variations of Passive Biaxial Mechanical Properties of Rat Myocardium. Preprints, 2021. 2021090244.

17. He, H., et al., A comparative study of 85 hyperelastic constitutive models for both unfilled rubber and highly filled rubber nanocomposite material. Nano Materials Science, 2021.

18. Holzapfel, G.A., et al., Determination of layer-specific mechanical properties of human coronary arteries with nonatherosclerotic intimal thickening and related constitutive modeling. American Journal of Physiology-Heart and Circulatory Physiology, 2005. 289(5): p. H2048-H2058.

19. Nemavhola, F., H.M. Ngwangwa, and T. Pandelani, An Investigation of Uniaxial Mechanical Properties of Excised Sheep Heart Muscle Fibre-Fitting of Different Hyperelastic Constitutive Models. Preprints, 2021. 2021080566 (doi: 10.20944/preprints202108.0566.v1).

20. Holzapfel, G.A. and R.W. Ogden, Constitutive modelling of passive myocardium: a structurally based framework for material characterization. Philosophical Transactions of the Royal Society A: Mathematical, Physical and Engineering Sciences, 2009. 367(1902): p. 34453475.

21. Ferruzzi, J., D.A. Vorp, and J. Humphrey, On constitutive descriptors of the biaxial mechanical behaviour of human abdominal aorta and aneurysms. Journal of the Royal Society Interface, 2011. 8(56): p. 435-450.

22. Nemavhola, F., Detailed structural assessment of healthy interventricular septum in the presence of remodeling infarct in the free wall-A finite element model. Heliyon, 2019. 5(6): p. e01841.

23. Ngwangwa, H.M. and F. Nemavhola, Evaluating computational performances of hyperelastic models on supraspinatus tendon uniaxial tensile test data. Journal of Computational Applied Mechanics, 2021. 52(1): p. 27-43.

24. Nemavhola, F., Fibrotic infarction on the LV free wall may alter the mechanics of healthy septal wall during passive filling. Bio-medical materials and engineering, 2017. 28(6): p. 579599.

25. Nemavhola, F., Mechanics of the septal wall may be affected by the presence of fibrotic infarct in the free wall at end-systole. International Journal of Medical Engineering and Informatics, 2019. 11(3): p. 205-225.

26. Javani, S., M. Gordon, and A.N. Azadani, Biomechanical properties and microstructure of heart chambers: A paired comparison study in an ovine model. Annals of biomedical engineering, 2016. 44(11): p. 3266-3283.

27. de Bortoli, D., et al. Hyperfit-curve fitting software for incompressible hyperelastic material models. in Proceedings of COBEM. 2011.

28. Chuong, C. and Y. Fung, Three-dimensional stress distribution in arteries. Journal of biomechanical engineering, 1983. 105(3): p. 268-274. 
29. Holzapfel, G.A., T.C. Gasser, and R.W. Ogden, A new constitutive framework for arterial wall mechanics and a comparative study of material models. Journal of elasticity and the physical science of solids, 2000. 61(1): p. 1-48.

30. Baek, S., et al., Theory of small on large: potential utility in computations of fluid-solid interactions in arteries. Computer methods in applied mechanics and engineering, 2007. 196(31-32): p. 3070-3078. 